\title{
Wearable Accelerometer Based Extended Sleep Position Recognition
}

\author{
Sarah Fallmann*, Rick van Veen*†, Liming Chen*, David Walker ${ }^{\dagger}$, Feng Chen* and Chengsheng Pan ${ }^{\ddagger}$ \\ ${ }^{*}$ School of Computer Science and Informatics, De Montfort University, Leicester, UK \\ Email: \{sarah.fallmann, liming.chen, feng.chen\}@dmu.ac.uk \\ ${ }^{\dagger}$ Philips Research UK, Cambridge, UK. Email: \{rick.van.veen, david.walker\}@philips.com \\ ‡Dalian University, Dalian, China. Email: pancs@sohu.com
}

\begin{abstract}
Sleep positions have an impact on sleep quality and therefore need to be further analyzed. Current research on position tracking includes only the four basic positions. In the context of wearable devices, energy efficiency is still an open issue. This research presents a way to detect eight positions with higher granularity under energy efficient constraints. Generalized Matrix Learning Vector Quantization is used, as it is a fast and appropriate method for environments with limited computation resources, and has not been seen for this kind of application before. The overall model trained on individuals performs with an averaged accuracy of $99.8 \%$, in contrast to an averaged accuracy of $83.62 \%$ for grouped datasets. Real world application gives an accuracy of around $98 \%$. The results show that energy efficiency will be feasible, as performance stays similar for lower sampling rate. This is a step towards a mobile solution which gives more insight in person's sleep behaviour.
\end{abstract}

\section{INTRODUCTION}

Human behaviour analysis plays a significant role in smart healthcare and assisted living [1], [6], [7]. Among them, sleep is an important subject for people of all age groups. The elderly and people suffering from sleep disturbances can particularly benefit from sleep monitoring systems. Current research on sleep quality attempts to build a real world sleep tracking system. This enables people to gain insight into their own sleeping behaviour. Sleep quality is influenced by different sleeping positions [15]. For example, there exists a relationship between sleep postures and sleep parameters such as waking episodes. Moreover, as sleep positions are independent from sleep stages [13], it is necessary for an effective sleep quality assessment to include both.

The four main sleep positions are supine, prone, left lateral, and right lateral, which can be measured during sleep while there is no movement. Postures with a higher granularity need to be considered, as leg positioning could lead to hip rotation resulting in back pain [8]. In general, motivations for sleep position tracking are often found in the prevention of pressure ulcers [17], [24] or sleep apnea [5]. Research has been done to monitor the four basic sleep postures taking into account simulated and real world data using different sleep position trackers. Those trackers can be grouped into three categories. The first category uses sensors installed in the bed [3], [10], [16], [17], [24]. The issue with this method is, that it cannot distinguish between the supine and prone positions. Furthermore, this is an immobile system as it requires sensors to be installed in or on the bed. The second category uses wearable devices [2], [14], [15], [21], which provide a mobile solution and are able to monitor different people in the same bed. A disadvantage is that the sensors have to be worn on the body, which can possibly lead to discomfort. The last category uses imaging [11]. Although, it can result in better position recognition, the main disadvantages of using imaging, e.g., cameras or kinect sensors, are the privacy issues people have and the inability of the system to accurately recognize positions through blankets [11].

As stated above, wearable devices are commonly used to track sleep positions. Two of the remaining issues are to find a robust and energy efficient method and track positions with finer granularity. In this study 3 -axis accelerometer data was collected using three Shimmer3 devices, worn on the ankles and the chest in both a simulated and real world setting. To improve the usability and comfort of wearable solutions there is a need for this type of sensor to be integrated into clothing [22]. Some integrated sensors for motion detection are already on the market like Enflux [9] or Xsens MVN [18]. This goes even further in [23], where a method is proposed that enables wearing sensor technologies directly on the skin. An approach using wearable devices can therefore become more practical.

Eight positions were tracked and classified using Generalized Matrix Learning Vector Quantization (GMLVQ) [19]. Similar to the k-nearest neighbours ( $\mathrm{kNN}$ ) algorithm, GMLVQ is a distance based classifier. However, it only computes the distance between a novel data pattern and the model's prototypes. Consequently, GMLVQ scales better, since the number of prototypes does not increase, when new data become available. This makes GMLVQ a fast and appropriate method for environments with limited computational resources. In this work the performance is tested during various experiments under normal and energy efficient settings. These experiments look at individual and grouped datasets. Moreover, results from lower sampling rate and fewer features are provided.

The concept presented here looks into the possibility for a mobile solution validated on real world data. In addition to the four basic positions, the presented research recognizes five more classes (overall eight positions plus the transition between them). Furthermore, experiments are conducted using GLMVQ to investigate the possibility of a more energy efficient system, by using the constructed model to reduce the number of features necessary.

The paper is structured in the following way. Section II discusses the related work. In Section III the approach for the 
sleep position recognition is described. Section IV presents the data analysis and their results which are discussed in Section V. Section VI concludes the conducted position recognition approach and describes possible future extension.

\section{RELATED WORK}

In this section the three main sleep position monitoring approaches discussed, are: sensors installed in and on the bed, wearable devices, and imaging sensors.

Pressure sensors integrated in the bed or bed sheets report generally a high level of accuracy (over 90\%) and suggest the possibility to detect body location and posture in the bed. For this approach different methodologies are used including: pressure amplitude value change embedded in a logistic regression model [3], pressure image analysis [12], [17], Bayesian classifier [10], and an algorithm combining normalization, Eigenspace projection, and a $\mathrm{kNN}$ classifier [24]. The approaches try to distinguish up to 13 different positions [16] showing difficulties with distinguishing the prone from the supine position. In the studies, data from a different number of subjects were collected. In most cases, data was collected in a simulated setting, from two [10], three [3], six [24], nine [16] and 20 [17] participants. Liu et al. [12] collected data from 14 subjects in a simulated, and three in a real world situation. In all those studies only Barsocchi et al. [3] addresses energy efficiency by keeping the sampling rate as low as possible, i.e., $10 \mathrm{~Hz}$.

The second approach uses wearable devices to gather data from 3-axis acceleration sensors.Nam et al. [14] developed a sleep quality calculation method including the sleep positions and stages, apnea, and sleep time. The four main sleeping poses are tracked from data gathered from a threeaxis accelerometer placed on the hip and evaluated with a portable diagnostic system. Barsocchi [2] includes a wearable device on the chest and three fixed environmental devices. Five positions (four main plus lateral in a $30 \%$ incline) are classified using the received signal strength measured among the fixed devices. Data was collected in a standard bedroom on two participants with $8 \mathrm{~Hz}$ sampling frequency. Extracted features include mean, standard deviation, skewness, and kurtosis when one receiver is used. Cross correlation is included when more than one sensor is used. Besides detecting the positions, the study also included the detection of motion versus nomotion. In all cases $\mathrm{kNN}$ always performs slightly better than Support Vector Machines. A 100\% accuracy is achieved for recognizing three of the five positions and $85 \%$ for the other two. Van Laerhoven et al. [21] uses tilt switches on the dominant wrist to be able to detect eight postures during the sleep. These postures include the four main positions plus four intermediate positions based on angle, e.g., left-prone. The go-to-bed and wake up time were analyzed using motion and ambient light. The data for sleep posture detection came from four subjects during nine nights. Their results show the limitations of the used devices, as some postures have the same tilt switch output. An average precision of $80 \%$ was achieved for three of the main positions, which are supine, left and right lateral and $73 \%$ for the extended postures. [25] presented an approach for a sleep quality measurement including position detection with an accelerometer placed on the chest giving an accuracy for the four basic positions of $99 \%$.

The third approach is based on imaging analysis techniques. Lee et al. [11] applied a kinect sensor hung over the bed to distinguish five different common sleep postures from 20 students. There are three limitations. Firstly, the system only works with people who do not use a blanket during the night. Secondly, prone and supine positions are not distinguishable. Thirdly, privacy issues arise when imaging device are used.

This paper addressed the following open issues using a wearable approach, which is less privacy sensitive and still mobile. Firstly, the number of positions detected in previous studies are in general limited to the four basic positions. These can be extended by positions with higher granularity giving more insight into sleep behaviour. To investigate this, the number of sensors is raised to three, to track the relevant lower limbs movement. Secondly, the performance under energy consumption restrictions is investigated, e.g., reduced sampling rate and number of features. Lastly, the existing research lacks a comparative study about differences of real world and simulated data.

\section{Sleep Position Recognition ApproACH}

This section describes the proposed sleep position recognition approach. This includes an general overview of the concept, how the data is collected, and a brief review of the GMLVQ model.

\section{A. General Overview}

Sleep position recognition can be divided into three parts, see Fig. 1. Firstly, the collected accelerometer data from the chest and legs is classified using the acceleration-moving variance method [20], into stable and non-stable time windows. Other methodologies to detect stable states exist, but choosing this one has the advantage that no other sensor data needs to be included, e.g., gyroscopes or magnetometers. Secondly, the stable windows are reduced to a nine dimensional data vector by calculating the mean value for each of the three accelerometer axes. Where, for example, $\mathrm{X}_{\text {Chest }}$ denotes the value of the accelerometer in the $X$ axis. In the last step the GMLVQ model classifies this feature vector in one of the pretrained classes, see Section III-B.

\section{B. Data Collection}

Accelerometer data was collected from six healthy participants, with a sampling frequency of $52 \mathrm{~Hz}$. Four males and two females, in one of the two age groups 28-33(three male, one female) and 55-58(one female, one male), participated 


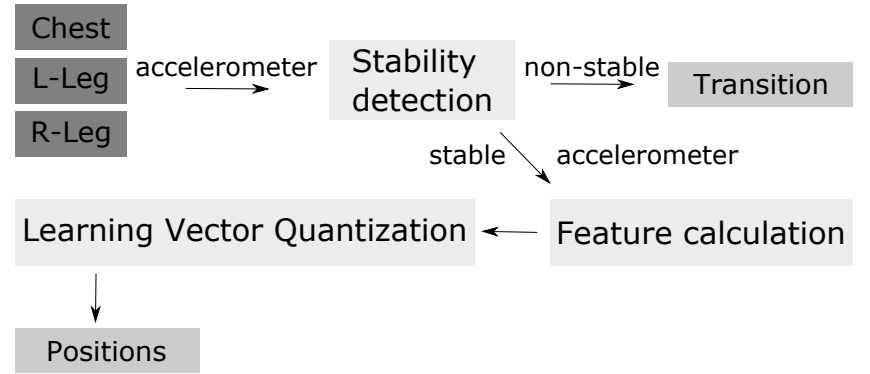

Fig. 1. Concept of sleep position detection with three Shimmer3 devices applied on the chest and ankles.

in a simulated scenario. Furthermore, the data collection was extended for two of the participants (one female, one male) to a real world setting, where they were continuously monitored during one night.

The simulated scenario was executed under the most natural circumstances possible. The participants were in a bed with a pillow and covered by a blanket. They were asked to wear three Shimmer3 sensors, one on each of their ankles and one on the chest, as illustrated in Fig. 2(a). Note that the sensors are all placed in the same orientation for all participants. The participants were told to assume each of the ten most common sleeping positions [13] for at least 25 seconds in nine cycles. One cycle consisted of ten positions, and no transition was performed twice. Figure 2(b) illustrates the positions which are described below.

Supine position laying on the back with straight legs $(S)$. Two variations were monitored including right leg bent $\left(S_{R L B}\right)$, and left leg bent $\left(S_{L L B}\right)$.

Prone position laying on the stomach $(P)$.

Left lateral position laying on the left side with either legs together $\left(L_{L T}\right)$ or right leg bent $\left(L_{R L B}\right)$.

Right lateral position laying on the right side with either legs together $\left(R_{L T}\right)$ or left leg bent $\left(R_{L L B}\right)$.

Note that no sensors on the arms are necessary, as the arms are bent in a similar way in all of the positions. The data was annotated using a mobile device, controlled by a researcher, marking the start and end moment of a position.

In the real world scenario two subjects were continuously monitored for around seven hours using the same sensor placements as in the simulated scenario. Additionally, the participants were recorded to allow later annotation of the stable periods, meaning the time windows where the participant was not moving. Those periods were labeled with the positions of interest and detected by computing the acceleration-moving variance $(\alpha)$ on all the available acceleration data [20]. The decision whether a state was stable, was determined by using a threshold, i.e., $\alpha<0.5$, which was found empirically. The three classes of the right and left lateral positions are fused into two classes, as legs bent $(R / L)_{L B}$ and legs straight $(R / L)_{L S}$ cannot be distinguished by the approach presented here. This

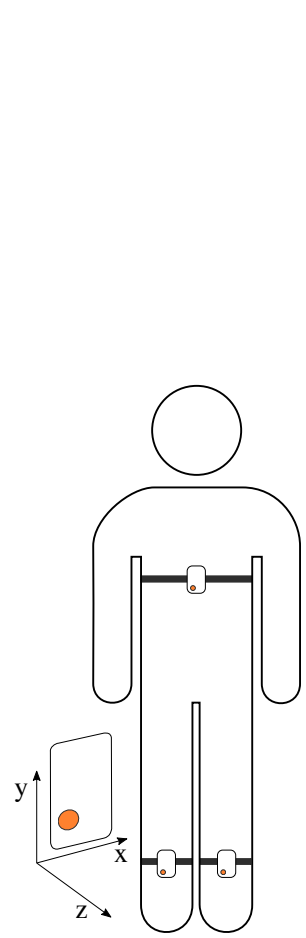

(a)
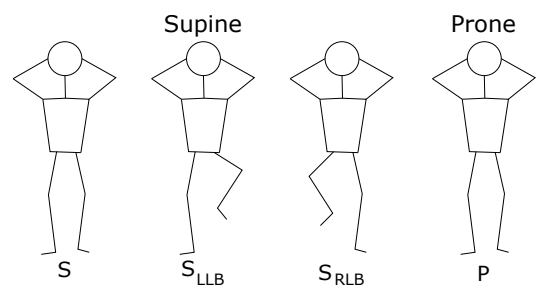

Right Lateral

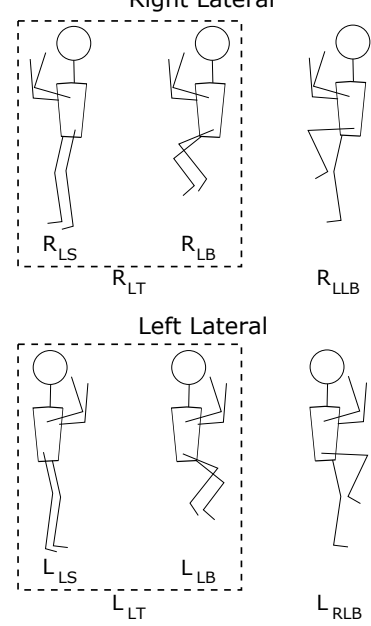

(b)
Fig. 2. (a) The placement of the sensors on the body. (b) Illustrates the monitored positions. The dotted lines indicate the two positions that are merged into one, e.g., $R_{L S}$ (legs straight) with $R_{L B}$ (legs bent) to $R_{L T}$.

merging is illustrated in Fig. 2(b) by the square boxes in dotted line.

The raw accelerometer data were processed by dividing it into windows of the size of the sampling rate, with an $50 \%$ overlap. The sensor values in the windows were averaged to obtain the final feature vector for every window. To address the aspect of energy efficiency the dataset was down-sampled to approximately $6 \mathrm{~Hz}$ for certain experiments.

\section{Classification Algorithm}

This section gives a brief review of the Generalized Matrix Learning Vector Quantization (GMLVQ) [19]. GMLVQ is an adaptive distance based supervised learning method. The model is constructed during the training phase where typical representations (prototypes) are found for each class. In addition to the prototypes, the GMLVQ algorithm constructs a relevance matrix $(\Lambda)$ that transforms the data in a way such that classes become better distinguishable. Considering a $n$ dimensional feature vector $\vec{\xi}$, and a prototype $\vec{\omega}$, the adaptive distance measure becomes

$$
d^{\Lambda}(\vec{\omega}, \vec{\xi})=(\vec{\xi}-\vec{\omega})^{T} \Lambda(\vec{\xi}-\vec{\omega}),
$$

where $\Lambda$ is a $n \times n$ matrix which accounts for the correlations between the features and rotation of the axes, by weighing every pair of features, resulting in a more robust performance [4], [19]. GMLVQ provides an intuitive model that can help 
to understand the data, e.g., the prototypes exist in the same space as the data and can therefore be directly interpreted. Moreover, the values of the diagonal and off-diagonal of $\Lambda$ can be interpreted as the relevance of a single feature and every combinations of two features respectively [19].

\section{DATA AnAlysis AND RESUlts}

This section describes the analyses of simulated and real world data to provide insight into personalized and general models. The individual GMLVQ model is trained using the data from one person and tested by repeating 10 -fold cross-validation, 20 times. The general model trains in a leave-one-person out manner using a grouped dataset of all other participants averaged over 20 runs. Additional experiments are done under simulated energy consumption constraints, by lowering the sampling rate, and reducing the number of sensors.

\section{A. Sleep Position Model Generalization}

The analyses discussed here are designed to provide insight into possible generalization of the model.

a) Individual Models: A separate repeated cross-validation analysis is executed for each of the participants. The averaged accuracy of these validation runs is $99.8 \%$.

b) General Model: The general model is based on a leaveone-person-out approach. In other words, the system tests on one person and trains on all the other participants. The averaged confusion matrix is given in Table I. The confusion

TABLE I

AVERAGED CONFUSION MATRIX FOR THE GENERAL MODEL.

\begin{tabular}{lcccccccc|c} 
& $\mathrm{S}$ & $\mathrm{S}_{L L B}$ & $\mathrm{~S}_{R L B}$ & $\mathrm{R}_{L T}$ & $\mathrm{R}_{L L B}$ & $\mathrm{~L}_{L T}$ & $\mathrm{~L}_{R L B}$ & $\mathrm{P}$ & Recall \\
\hline $\mathrm{S}$ & 94.3 & 2.04 & 3.64 & 0 & 0 & 0 & 0 & 0 & 91.35 \\
$\mathrm{~S}_{L L B}$ & 3.94 & 96.06 & 0 & 0 & 0 & 0 & 0 & 0 & 96.24 \\
$\mathrm{~S}_{R L B}$ & 1.83 & 0 & 98.17 & 0 & 0 & 0 & 0 & 0 & 98.20 \\
$\mathrm{R}_{L T}$ & 0 & 0 & 0 & 81.8 & 18.2 & 0 & 0 & 0 & 83.30 \\
$\mathrm{R}_{L L B}$ & 0 & 0 & 0 & 53.41 & 44.65 & 0 & 0 & 1.93 & 42.32 \\
$\mathrm{~L}_{L T}$ & 0 & 0.85 & 0 & 0 & 0 & 91.7 & 7.45 & 0 & 91.75 \\
$\mathrm{~L}_{R L B}$ & 0 & 0 & 0 & 0 & 0 & 30.87 & 67.35 & 1.78 & 67.37 \\
$\mathrm{P}$ & 0 & 0 & 0 & 0 & 7.86 & 0 & 0 & 92.14 & 88.75 \\
\hline
\end{tabular}

Prec. $95.8796 .1796 .3379 .3063 .04 \quad 88.7171 .13 \quad 96.75 \mid 83.62$

matrix in Table I shows an overall accuracy of $83.62 \%$, but also that the model has difficulties with the lateral positions. Furthermore, the results display a number of misclassifications between, e.g., the lateral and prone position. A second analysis shows the increase of performance when the two lateral subclasses for right and left lateral respectively, meaning $\left(R_{L T}\right.$ and $\left.R_{L L B}\right)$ and $\left(L_{L T}\right.$ and $\left.L_{R L B}\right)$ are fused together. This results in six positions and $98.31 \%$ accuracy.

\section{B. Mechanisms to Improve Energy Efficiency}

In this section the performance of the model is analyzed to gain insight into the possibility of reducing sampling rate and the number of sensors and features used in order to increase energy efficiency.

a) Reduced Sampling Rate: For each of the individual models a dataset, using a sampling frequency of around $6 \mathrm{~Hz}$, was created by down-sampling the original data. This caused no reduction of performance in the different individual models.

b) Reduced Number of Sensors: A further experiment is executed using only the sensors that were placed on the ankles, resulting in an accuracy of $79.71 \%$ for eight positions and $96.08 \%$ for six positions, trained as in the generalized approach. In contrast, the individual models show an output of around $99.8 \%$ accuracy, which is a similar result as for three sensors.

c) Feature Selection: Using the relevance matrix, $\Lambda$, provided by the GMVLQ model, a feature selection can be done. The averaged relevance values of the features are included in Fig. 3(a). Together with the top three ranking, shown in Fig. 3(b), the three most relevant features are: $X_{\text {Chest }}, Z_{\text {Left }}$, and $\mathrm{Z}_{\text {Right }}$. For the individual and the general model the order of relevance is illustrated in Table II. Note that all of the combinations are unique.

Applying the experiments on the reduced data shows that the combination of the three best ranked features gives an approximate accuracy of $78 \%$ for the general model.

TABLE II

FEATURE RANKING FOR THE INDIVIDUAL AND GENERAL MODELS.

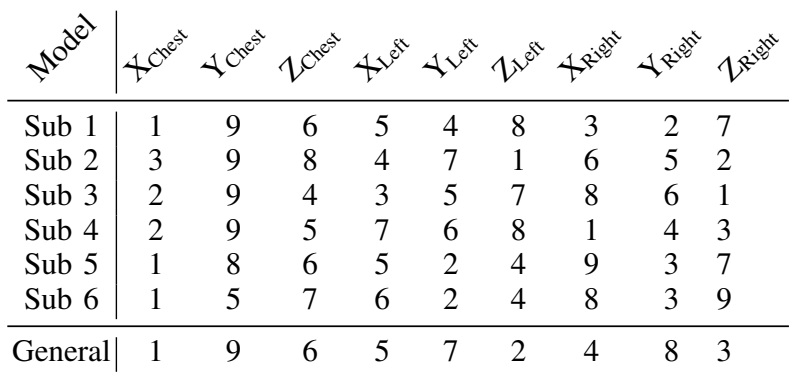

\section{Application in the Real World}

The real world experiment applied the general model on two real world datasets each containing data of one sleep cycle.

Participant 1 changes between six detectable positions during one night, $S, S_{L L B}, S_{R L B}, R_{L T}, R_{L L B}, L_{L T}$. When the general model is applied, it results in an averaged accuracy of $98 \%$. When only using the three best features in the general model the accuracy decreases to $89.5 \%$. In comparison the individual model gives an averaged accuracy of $58.39 \%$.

Participant 2 changes during the night between the same six positions as participant one. The general model gives an averaged accuracy of $97.48 \%$. When only using the three best features the accuracy decreases to $95.53 \%$. The individual model fails in this experiment. 


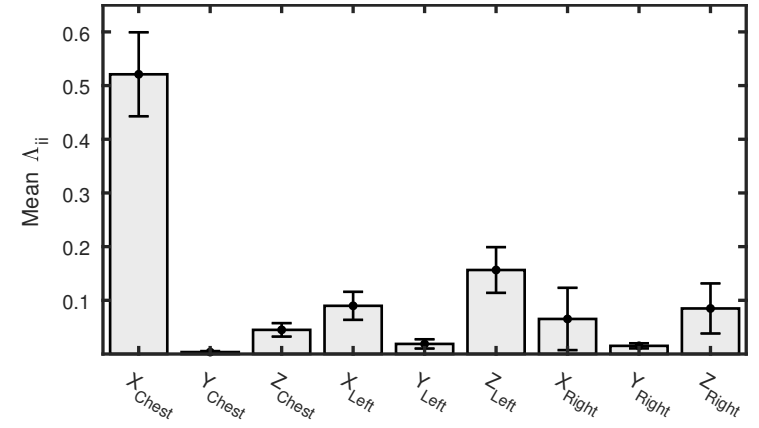

(a)

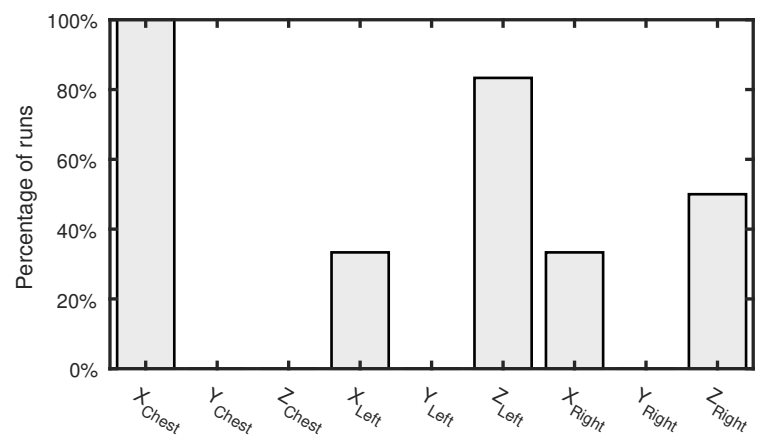

(b)

Fig. 3. (a) Averaged relevance matrix diagonal and standard deviation for every feature. (b) Percentage a feature was in the top 3 most relevant features of the 20 times 10 -fold cross-validation.

\section{DISCUSSION}

\section{A. Sleep Position Model Generalization}

a) Individual Model: The personalized model shows a near perfect performance on the simulated data. This suggests that the individuals are consistent in their positioning during the experiment. The reason for not having a perfect score is most probably caused by inaccurate annotations, leaking some of the transitions into the training data. A limitation of this approach is that it will need to be retrained for every individual, which requires an annotated dataset, making it impractical. To solve this an unsupervised learning method which can handle unlabeled datasets could be implemented. The reason why an individual model is investigated comes from a privacy perspective, as in this way no personal information of others is shared in the model.

b) General Model: A general model provides a prototype which does not need any data annotation and collection by an individual. It gives the possibility to provide an off-theshelf prototype with reasonable but limited results. These limitations are caused by representing the general position information. This can lead to misclassification as positions have an individual component. The model can likely be improved in three possible ways. Firstly, by decreasing the positions tracked to six. Secondly, by training the model with a small new collected individual annotated dataset. Thirdly, the misclassification between lateral and prone positions could potentially be improved using a rule-based approach dividing the four main positions, followed by training individual models for the sub-classes with higher granularity, e.g. $L_{T}$ and $L_{R L B}$.

\section{B. Mechanisms to Improve Energy Efficiency}

a) Feature selection: The feature selection shows that the general model can classify the positions with fewer features. A trade-off can be made, between accuracy and the need for computational power.

Table II displays the individuality of different positions, which means that individual information from subjects is relevant while training and testing the model. This also implies the possibility of distinguishing the people based on their model. This can be the reason why the general model is less accurate than the individual models, for some of the subjects.

b) Reduced Sampling Rate: To address the aspect of energy efficiency the sampling rate was reduced. Despite this reduction, the same amount of information of the positions was still available in the stable state data, resulting in similar high performance. This emphasizes the possibility for an energy efficient solution, e.g., with a dynamic sampling rate, meaning low rate during stable states (positions) and high during movement (transitions).

c) Reduced Number of Sensors: As a high number of sensors is undesirable, reducing the number of sensors was investigated. The results show that only using the leg sensors reduce the performance for the general model, but keeps the high performance for the individual models. This is caused by the individual preference and consistency of how to assume the positions.

\section{Application in the Real World}

The real world data experiment demonstrates that the general model performs accurately for the positions the participants adopt during the night, even though the general model is not trained on the participant real world data. In contrast, the individual model fails, leading to the conclusion that the simulated data does not represent the real world scenario in general. For an accurate position detection system it seems therefore more appropriate to use a general model which is trained on a subgroup of people and thereby captures the variability in the positions. For energy efficiency reasons a model was trained using only three of the best features, resulting in similar high accuracy. This provides a model which can work with only three 1 -axis accelerometers.

The classification gives appropriate results for most sleep positions, but has more difficulties when there is no difference in orientation between the positions. This is the case for the lateral positions including $(R / L)_{L T}$ and $(R / L)_{(L / R) L B}$. This is because accelerometers cannot capture the relative position in space, but only the orientation of the sensors. To distinguish all of the three higher granularity lateral positions in Fig. 2(b) a numerical integration concept is proposed in Section VI. 


\section{CONClusion And Future Work}

It has been shown that by using the accelerometers in Shimmer3 devices, eight specific positions can be detected even under a low sampling rate. Furthermore, the study has shown that the number of sensors can be reduced for just a small cost in performance. The general model appeared to be the best option for a real world application, better capturing the variability of the positions. By including these findings into a sleep quality assessment, sleep behaviour can likely be better understood.

Future work will integrate the sleep position recognition in a sleep quality representation. Supplementary position information could help users to self-manage their sleep better. The presented system can easily be applied for bed exit detection. Additionally, the current list of tracked positions will be extended. Positions, such as the four positions that were merged (shown in Fig. 2(b)), were hard to distinguish using orientation information only. This can possibly be solved by including the position of the feet into the calculation, this information can be extracted by integrating the accelerometer data twice. The limitation of the concept is, that numerical integration increases small errors drastically. Therefore, integration should be conducted during a short period of time. This is the case during the real world monitored transitions, where the average transition time was measured to be around three seconds.

\section{ACKNOWLEDGEMENT}

This project has received funding from the European Union's Horizon 2020 research and innovation program under the Marie Skłodowska-Curie grant agreement No 676157.

\section{REFERENCES}

[1] G. Azkune, A. Almeida, D. López de Ipiña, and L. Chen. Extending knowledge-driven activity models through data-driven learning techniques. Expert Systems with Applications, 42(6):3115-3128, 2015.

[2] P. Barsocchi. Position recognition to support bedsores prevention. IEEE Journal of Biomedical and Health Informatics, 17(1):53-59, 2013.

[3] P. Barsocchi, M. Bianchini, A. Crivello, D. L. Rosa, F. Palumbo, and F. Scarselli. An unobtrusive sleep monitoring system for the human sleep behaviour understanding. In 7th IEEE International Conference on Cognitive Infocommunications (CogInfoCom), pages 91-96, Oct 2016.

[4] M. Biehl, P. Schneider, D. Smith, H. Stiekema, A. Taylor, B. Hughes, C. Shackleton, P. Stewart, and W. Arlt. Matrix relevance LVQ in steroid metabolomics based classification of adrenal tumors. In M. Verleysen, editor, 20th European Symposium on Artificial Neural Networks (ESANN 2012), pages 423-428. d-side publishing, 2012.

[5] R. D. Cartwright. Effect of Sleep Position on Sleep Apnea Severity. Sleep, 7(2):110-114, 1984

[6] L. Chen, C. Nugent, and G. Okeyo. An ontology-based hybrid approach to activity modeling for smart homes. IEEE Transactions on HumanMachine Systems, 44(1):92-105, Feb 2014.

[7] L. Chen, C. D. Nugent, J. Biswas, and J. Hoey, editors. Activity Recognition in Pervasive Intelligent Environments, volume 4 of Atlantis Ambient and Pervasive Intelligence. Atlantis, Amsterdam, 2011.
[8] M. T. Cibulka and J. Threlkeld-Watkins. Patellofemoral pain and asymmetrical hip rotation. Physical Therapy, 85(11):1201-1207, 2005.

[9] Enflux. Motion capture clothing for creators and developers. Online, 2017. Available at https://www.getenflux.com/. Accessed: 30-06-2017.

[10] C.-C. Hsia, Y.-W. Hung, Y.-H. Chiu, and C.-H. Kang. Bayesian classification for bed posture detection based on kurtosis and skewness estimation. In HealthCom 2008 - 10th International Conference on e-health Networking, Applications and Services, pages 165-168, July 2008.

[11] J. Lee, M. Hong, and S. Ryu. Sleep Monitoring System Using Kinect Sensor. International Journal of Distributed Sensor Networks, pages 1-9, January 2015.

[12] J. J. Liu, W. Xu, M. C. Huang, N. Alshurafa, M. Sarrafzadeh, N. Raut, and B. Yadegar. Sleep posture analysis using a dense pressure sensitive bedsheet. Pervasive and Mobile Computing, 10:34-50, 2014

[13] J. De Lorrain, D.; Koninck. Sleep Position and Sleep Stages: Evidence of Their Independence. Clinical Medicine - Sleep, 21(4), June 1998.

[14] Y. Nam, Y. Kim, and J. Lee. Sleep monitoring based on a tri-axial accelerometer and a pressure sensor. Sensors (Switzerland), 16(5):1-14, 2016.

[15] A. Nojiri, C. Okumura, and Y. Ito. Sleep Posture Affects Sleep Parameters Differently in Young and Senior Japanese as Assessed by Actigraphy. Health, 6(21):2934-2944, December 2014.

[16] S. Ostadabbas, M. Baran Pouyan, M. Nourani, and N. Kehtarnavaz. Inbed posture classification and limb identification. IEEE 2014 Biomedical Circuits and Systems Conference, BioCAS 2014 - Proceedings, pages 133-136, 2014.

[17] M. B. Pouyan, S. Ostadabbas, M. Farshbaf, R. Yousefi, M. Nourani, and M. D. M. Pompeo. Continuous eight-posture classification for bedbound patients. In 2013 6th International Conference on Biomedical Engineering and Informatics, pages 121-126, Dec 2013.

[18] D. Roetenberg, H. Luinge, and P. Slycke. Xsens MVN: Full 6DOF Human Motion Tracking Using Miniature Inertial Sensors. Technical report, xsens, 2009

[19] P. Schneider, M. Biehl, and B. Hammer. Adaptive Relevance Matrices in Learning Vector Quantization. Neural computation, 21(12):3532-3561, 2009.

[20] I. Skog, P. Handel, J. O. Nilsson, and J. Rantakokko. Zero-Velocity Detection; An Algorithm Evaluation. IEEE Transactions on Biomedical Engineering, 57(11):2657-2666, Nov 2010.

[21] K. Van Laerhoven, M. Borazio, D. Kilian, and B. Schiele. Sustained logging and discrimination of sleep postures ith low-level, wrist-worn sensors. Proceedings - International Symposium on Wearable Computers, ISWC, pages 69-76, 2008.

[22] Z. Wang, Z. Yang, and T. Dong. A review of wearable technologies for elderly care that can accurately track indoor position, recognize physical activities and monitor vital signs in real time. Sensors, 17(2):341, 2017.

[23] S. Xu, Y. Zhang, L. Jia, K. E. Mathewson, K. I. Jang, J. Kim, H. Fu, X. Huang, P. Chava, R. Wang, S. Bhole, L. Wang, Y. J. Na, Y. Guan, M. Flavin, Z. Han, Y. Huang, and J. A. Rogers. Soft microfluidic assemblies of sensors, circuits, and radios for the skin. Science, 344:70$74,2014$.

[24] R. Yousefi, S. Ostadabbas, M. Faezipour, M. Farshbaf, M. Nourani, L. Tamil, and M. Pompeo. Bed posture classification for pressure ulcer prevention. Proceedings of the Annual International Conference of the IEEE Engineering in Medicine and Biology Society, EMBS, pages 71757178,2011

[25] Z. Zhang and G. Yang. Monitoring cardio-respiratory and posture movements during sleep: What can be achieved by a single motion sensor. In 12th IEEE International Conference on Wearable and Implantable Body Sensor Networks, BSN 2015, Cambridge, MA, USA, pages 1-6, June 2015. 